\title{
MULTI-CRITERIA GROUP DECISION MAKING WITH HETEROGENEOUS INFORMATION BASED ON IDEAL POINTS CONCEPT
}

\author{
DING-HONG PENG ${ }^{1,2} *$, CHANG-YUAN GAO ${ }^{1}$, LI-LI ZHAI ${ }^{1}$ \\ ${ }^{1}$ School of Management, Harbin University of Science and Technology \\ Harbin 150040, PR China \\ ${ }^{2}$ Institute of Quality Development, Kunming University of Science and Technology \\ Kunming 650093, PR China \\ *E-mail: pengdinghong2009@163.com \\ Received 20 December 2010 \\ Accepted 28 October 2011
}

\begin{abstract}
This paper presents a flexible multi-criteria group decision making method based on ideal points concept, which can be used to deal with heterogeneous information(numerical, interval valued and linguistic variable with different granularity and/or semantic)and reflect the Decision Makers' different decision attitudes. The heterogeneous information is homogenized firstly into linguistic variable characterized fuzzy number. To simplify the computations and improve the comprehensibility, the homogenized information is further transformed to the continue linguistic terms set. A new relative closeness measure based on ordered weighted distance is introduced to consider the decision Makers' different decision attitudes. A numerical experiment is used to illustrate the feasibility of the proposed method.
\end{abstract}

Keywords: multi-criteria group decision making (MCGDM), ideal points, heterogeneous information, information uniform, ordered weighted distance (OWD) measure, decision attitude.

\section{Introduction}

TOPSIS (Technique for Order Performance by Similarity to Ideal Solution), one of the most widely used multi-criteria decision making (MCDM) method, was first presented by Hwang and Yoon (1981) ${ }^{1}$ for solving a MCDM problem. The basic idea of the TOPSIS method is that the chosen alternative should have the shortest distance from the positive ideal solution (PIS) and the farthest distance from the negative ideal solution (NIS). TOPSIS cherishes such features $a^{2}$ : (i) a sound logic that represents the rationale of human choice; (ii) a scalar value that accounts for both the best and worst alternatives simultaneously; (iii) a simple computation process; and (iv) the performance measures of all alternatives on attributes can be visualized on a polyhedron, at least for any two dimensions.

With the decision environment becomes much more complexity, many decision making problems call for a collaborative efforts of experts, and usually, the fact that 
the uncertainty of experts' knowledge. Hence, many efforts have extended TOPSIS to various group decision situations in which the decision information is fuzzy, interval-valued or intuitionstic fuzzy, and so on, to fit real-world, for example, Chen $(2000)^{3}$ extended TOPSIS to develop a methodology for solving multi-person multi-criteria decision-making problem in fuzzy environment, Jahanshahloo et al. (2006) ${ }^{4}$ extended TOPSIS with interval number, Wang and Elhag (2006) proposed a nonlinear programming solution procedure to extend TOPSIS in fuzzy environment based on alpha level sets. Wang and Lee (2007) ${ }^{6}$ generalized TOPSIS in a fuzzy environment, proposed Up and Lo operators instead of usual max and min operations, which satisfied the partial ordering relation on fuzzy numbers, to find the ideal solution and negative ideal solution. Chen and Tzeng (2004) $)^{7}$ combined grey relation and TOPSIS to select an expatriate host country, where fuzzy AHP is used to determine subjective judgments weights, the grey relation model is combined to TOPSIS, and sugeno integral is used to consider the interaction among critera. García-Cascales and Lamata $^{8}$ incorporated descriptive, linguistic, ordinal variables, and presented the pseudo-distances between fuzzy numbers to TOPSIS method. Chen and Lee $(2010)^{9}$ presented an interval type-2 fuzzy TOPSIS method to handle fuzzy multiple attributes group decision-making problems based on interval type-2 fuzzy sets. Li $(2010)^{10}$ constructed a new nonlinear programming methodology based on TOPSIS to deal with multiattribute decision making with interval-valued intuitionistic fuzzy sets. Considering the interaction among attributes cannot be ignored, $\mathrm{Hu}$ $(2008)^{11}$ proposed choquet integral-based TOPSIS method, the choquet integral-based distance measure is used to compute the distances of alternatives to the PIS and the NIS. Additionally, Zanakis et al. (1998) ${ }^{12}$ verified that TOPSIS has the fewest rank reversals among the eight methods of MCDM by simulation comparison.

As reviewed above, most of the proposals for solving decision making problems with multiple experts are focused on the cases where all the experts express their opinions by means of values from the same type, either real values, interval values or linguistic labels in the same linguistic term set. However, due to the fact that each decision maker has his/her own unique characteristics with regard to knowledge, skills, experience and personality, the different decision makers tend to utilize different ways (such as real values, interval values and linguistic labels) to present their cognitions of information. The approaches mentioned above can not satisfy the practical situation. In this paper, we will propose a novel multiple criteria group decision-making methodology based on ideal points that work well with heterogeneous information (real values, interval values, linguistic labels in different linguistic term sets represented by different semantic), which allows the decision makers express free their assessments according to their preferences. Moreover, in view of the decision situations should be different when facing different decision problems, and then, the decision attitudes of decision makers are also different. In the decision framework we will construct can also offer decision makers the possibility of expressing their decision attitudes according to their interests and the nature of decision problems to increase flexibility of decision analysis.

The rest of this paper is organized as follows. Section 2 is devoted to briefly review some foundational concepts and definitions on linguistic variable. In Section 3 we introduce the proposed method in detail. In Section 4 an illustrative example is included to demonstrate the process of the proposed method, and the paper is concluded in Section 5.

\section{Preliminaries}

For the sake of clarity, some basic concepts and definitions on linguistic variable are stated as follows.

\subsection{Linguistic term set}

Definition $\mathbf{1}^{13}$. A linguistic variable is one whose values are not numbers but rather words or sentences in a natural or artificial language, i.e., linguistic terms.

The concept of linguistic variable is very useful in dealing with situations that are too complex or too illdefined to reasonably describe using conventional quantitative expressions.

Definition $2^{14}$. Let $S=\left\{s_{i}\right\}, i \in H=\{0, \ldots, g\}$, be a finite and totally ordered term set with odd cardinal, any term $s_{i}$ represents a possible value for a linguistic real variable, where the middle term represents an 
uncertainty of "approximately 0.5 " and the remaining terms are placed symmetrically around it.

Definition 3. The linguistic term set satisfies the following additional characteristics: ${ }^{14}$

(i) The set is ordered: $s_{i} \geq s_{j}$ if $i \geq j$.

(ii) There is a negation operator: $\operatorname{Neg}\left(s_{i}\right)=s_{j}$, $j=T-i(T+1$ is the cardinality).

(iii) Maximization operator: $\max \left(s_{i}, s_{j}\right)=s_{i}$ if $s_{i} \geq s_{j}$.

(iv) Minimization operator: $\min \left(s_{i}, s_{j}\right)=s_{i}$ if $s_{i} \leq s_{j}$.

For example, a set of seven terms, $S$, could be given as follows: $S=\left\{s_{0}=\right.$ none, $s_{1}=$ very low,$s_{2}=$ low,$s_{3}=$ medium, $s_{4}=$ high,$s_{5}=$ very high, $s_{6}=$ perfect $\}$.

\subsection{Trapezoidal fuzzy number}

Definition 4. For a trapezoidal fuzzy number $\tilde{A}=(a, b, c, d), a \leq b \leq c \leq d$, its membership function is

$$
\mu_{\tilde{A}}(x)=\left\{\begin{array}{cc}
0, & x<a \\
(x-a) /(b-a), & a \leq x \leq b \\
1, & b \leq x \leq c . \\
(x-d) /(c-d), & c \leq x \leq d \\
0, & x>d
\end{array}\right.
$$

This parametric representation is achieved by the 4 tuple $(a, b, c, d)$, where $b$ and $c$ indicate the interval in which the membership value is $1, a$ and $d$ indicate the left and right limits of the definition domain of the trapezoidal membership function ${ }^{13}$. Specifically, when $b=c, \tilde{A}$ is reduced to triangular fuzzy number $(a, b=c, d)$, when $a=b, c=d, \tilde{A}$ is reduced to interval number $(a=b, c=d)$, when $a=b=c=d, \tilde{A}$ is reduced to crisp number. On the contrary, triangular fuzzy number, interval number, crisp number can be regarded as the very special cases of trapezoidal fuzzy number.

In generally, each linguistic term $S_{i} \in S_{T}$ is characterized by trapezoidal fuzzy number $\left(a_{i}, b_{i}, c_{i}, d_{i}\right), a_{i}, b_{i}, c_{i}, d_{i} \in[0,1], i \in\{0,1, \ldots, g\}$ in the interval of $[0,1]$. For example, we may assign the semantics of Fig. 1 to a linguistic term set of seven terms. In linguistic decision making procedure, different DMs have different uncertainty degrees on decision problem, they usually use several linguistic term sets with different granularity of uncertainty to conduct decision making. The use of several linguistic term sets gives decision makers more flexibility. The more linguistic terms used, the smaller the range that each linguistic term cover.

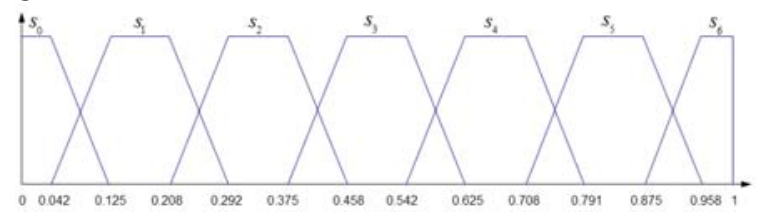

Fig. 1. A set of seven terms with their semantics.

In the process of the linguistic information proceeding, however, some results may not exactly match any linguistic terms in $S$. To preserve all the given information, $\mathrm{Xu}^{15}$ extended the discrete term set $S$ to a continuous term set $\bar{S}=\left\{s_{\alpha} \mid \alpha \in[0, q]\right\}$, whose elements meet $s_{\beta}>s_{\alpha}$ if $\beta>\alpha$, and where, if $s_{\alpha} \in S$, then we call $s_{\alpha}$ the original term, otherwise, we call $s_{\alpha}$ the virtual term, and $q$ is a large enough positive integer. In general, the decision maker uses the original terms to evaluate criteria and alternatives, and the virtual terms can only appear in calculation.

Definition $5^{16}$. Let any two linguistic labels $s_{\alpha}, s_{\beta} \in \bar{S}$, the primary algebraic operations are shown as follows:

$$
\begin{aligned}
& s_{\alpha} \oplus s_{\beta}=s_{\alpha+\beta} . \\
& \lambda s_{\alpha}=s_{\lambda \alpha}, \lambda \in[0,1] . \\
& d\left(s_{\alpha}, s_{\beta}\right)=|\alpha-\beta| .
\end{aligned}
$$

\section{The proposed method}

Assume that a group of $K$ decision-makers $D=\left\{D_{1}, \ldots, D_{k}\right\} \quad$ is responsible for selecting $m$ alternatives $A=\left\{A_{1}, \ldots, A_{m}\right\}$ under each of $n$ selection criteria $C=\left\{C_{1}, \ldots, C_{n}\right\}$. Criteria can be classified into benefit criteria $(B)$ and cost criteria $(C)$.

(1) Construct decision matrices

$$
D^{k}=\left[x_{i j}^{k}\right]_{m \times n}, k=1,2, \ldots, K .
$$

The assessments provided by DMs can be crisp values $x_{i j} \in R$, interval values $x_{i j}=\left[x_{i j}^{L}, x_{i j}^{U}\right]$, $\left(x_{i j}^{L} \leq x_{i j}^{U}, \quad x_{i j}^{L}, x_{i j}^{U} \in R\right)$, and linguistic values in different linguistic term sets represented by trapezoidal fuzzy function or triangular fuzzy functions $x_{i j} \in S=\left\{s_{0}, s_{1}, \ldots, s_{g}\right\}, 3 \leq g \leq 11^{14}$.

(2) Homogenize heterogeneous information

To homogenize the heterogeneous information, here we select a linguistic terms set, called basic linguistic 
term set $(\text { BLTS })^{17}$, to served as medium of unifying heterogeneous information.

\section{Select basic linguistic term set}

To maintain the uncertainty degrees associated to each one of the possible domains to be unified and the ability of discrimination to express the performance values, we select the linguistic term set with the maximum granularity as the basic linguistic term set (BLTS), $S_{T}=\left\{s_{0}, s_{1}, \ldots, s_{t}\right\}$.

Normalize numerical and interval-valued information.

Sometimes, the assessments with numerical and interval valued information can be expressed in different units. However, each linguistic term is characterized by trapezoidal or triangular fuzzy number in $[0,1]$ interval. To ensure compatibility among the numerical and interval valued evaluations with the linguistic ones, all the estimated numerical and interval values should be normalized firstly into a comparable scale.

The numeric value $x_{i j}$ is normalized into[0,1]interval by following:

$$
r_{i j}=x_{i j} / \sqrt{\sum_{i=1}^{m} x_{i j}^{2}}, i=1, \ldots, m, j=1, \ldots, n .
$$

The interval value $\left[x_{i j}^{L}, x_{i j}^{R}\right]$ is normalized by: ${ }^{4}$

$$
\begin{aligned}
& \bar{n}_{i j}^{L}=x_{i j}^{L} / \sqrt{\sum_{i=1}^{m}\left[\left(x_{i j}^{L}\right)^{2}+\left(x_{i j}^{R}\right)^{2}\right]}, i=1, \ldots, m, j=1, \ldots, n .(6) \\
& \bar{n}_{i j}^{R}=x_{i j}^{R} / \sqrt{\sum_{i=1}^{m}\left[\left(x_{i j}^{L}\right)^{2}+\left(x_{i j}^{R}\right)^{2}\right]}, i=1, \ldots, m, j=1, \ldots, n .
\end{aligned}
$$

Transform heterogeneous information into BLTS

Herrera et al. ${ }^{17}$ provided a transform function between linguistic term sets with different granularity. The idea of the transformation technique is to assign a degree of membership to every linguistic term in $S_{T}$ for each linguistic term being transformed. The degree of membership is computed by finding the interaction of two linguistic terms belonging to $S_{T}$ and $S$, respectively.

As mentioned above, numerical and interval values can be regarded as specifically trapezoidal fuzzy number, such as:

(1)When heterogeneous information is numerical $H^{N}$, then the normalized numerical value $r_{i j} \in[0,1]$ can be represented with membership function:

$$
\mu_{r_{i j}}(y)=\left\{\begin{array}{l}
1, y=r_{i j} \\
0, \text { otherwise }
\end{array} .\right.
$$

(2)When heterogeneous information is interval valued $H^{I}$, then the normalized interval value $I=\left[\bar{n}_{i j}^{L}, \bar{n}_{i j}^{R}\right] \subseteq[0,1]$ can be represented with membership function:

$$
\mu_{I}(y)=\left\{\begin{array}{l}
1, y \in I=\left[\bar{n}_{i j}^{L}, \bar{n}_{i j}^{R}\right] . \\
0, \text { otherwise }
\end{array} .\right.
$$

Hence, the transformation function also appropriately used to convert the normalized numerical and interval-valued assessments, the ranges of which belong to $[0,1]$, into BLTS.

Definition $6^{17,18,19}$. Let $H$ be heterogeneous information (numerical $H^{N}$, interval valued $H^{I}$ and linguistic $H^{L}$ in linguistic term set $\left.S=\left\{s_{0}, s_{1}, \ldots, s_{g}\right\}\right)$, and basic term set $S_{T}=\left\{s_{0}, s_{1}, \ldots, s_{t}\right\}(T \geq g)$, then the transformation function $\tau_{H S_{T}}$ is defined as:

$$
\begin{gathered}
\tau_{H S_{T}}: H \rightarrow F\left(S_{T}\right) \\
\tau_{H S_{T}}(H)=\left\{\left(s_{0}, \gamma_{0}\right), \ldots,\left(s_{t}, \gamma_{t}\right)\right\}, s_{i} \in S_{T}, \gamma_{i} \in[0,1](10) \\
\gamma_{i}=\max _{y} \min \left\{\alpha_{H}(y), \alpha_{s_{i}}(y)\right\}, \gamma_{i} \in[0,1] .
\end{gathered}
$$

where $F\left(S_{T}\right)$ is the set of fuzzy sets defined in $S_{T}=\left\{s_{0}, s_{1}, \ldots, s_{t}\right\} \quad$, and $\alpha_{H}(y), \alpha_{s_{i}}(y)$ are the membership functions of the fuzzy sets associated to the $H$ and $s_{i}$, respectively. The max-min operation is a classical tool for setting the matching degree between fuzzy sets.

Note that each numerical, interval-valued and linguistic value is expressed by means of a fuzzy set on the BLTS, $F\left(S_{T}\right)$. In order to clarify the transformation function, we provide the following examples.

Example 1. Let 0.78 be a numerical value and [0.25, $0.67]$ an interval value to be transformed into a fuzzy set in $S=\left\{s_{0}, s_{1}, s_{2}, s_{3}, s_{4}, s_{5}, s_{6}\right\}$, their uniformed values are:

$T(0.78)=\left\{\left(s_{0}, 0\right),\left(s_{1}, 0\right),\left(s_{2}, 0\right),\left(s_{3}, 0\right),\left(s_{4}, 0.14\right),\left(s_{5}, 0.86\right),(\right.$ $\left.\left.s_{6}, 0\right)\right\}$.

$T([0.25,0.67])=\left\{\left(s_{0}, 0\right),\left(s_{1}, 0.5\right),\left(s_{2}, 1\right),\left(s_{3}, 1\right),\left(s_{4}, 1\right),\left(s_{5}, 0\right.\right.$ ),$\left.\left(s_{6}, 0\right)\right\}$. Graphically, they are represented in Fig. 2 .

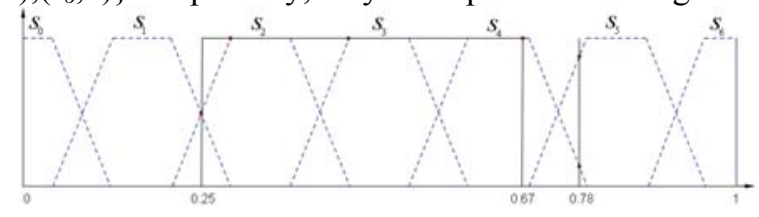

Fig. 2. The results of numerical value 0.78 and interval value $[0.25,0.67]$ are transformed into a fuzzy set in $S_{T}$.

Example 2. Let $s_{1}^{5}$ be the second linguistic term in the set with five terms represented by trapezoidal fuzzy number, $s_{3}^{5}$ be the forth linguistic term in the set with 
five terms represented by triangular fuzzy number. They are transformed into a fuzzy set in $S=\left\{s_{0}, s_{1}, s_{2}\right.$, $\left.S_{3}, s_{4}, s_{5}, s_{6}\right\}$ represented by trapezoidal fuzzy number. Their transformed values are: )$\}$

$$
T\left(s_{1}^{5}\right)=\left\{\left(s_{0}, 0.3\right),\left(s_{1}, 1\right),\left(s_{2}, 1\right),\left(s_{3}, 0.3\right),\left(s_{4}, 0\right),\left(s_{5}, 0\right),\left(s_{6}, 0\right.\right.
$$

$T\left(s_{3}^{\prime 5}\right)=\left\{\left(s_{0}, 0\right),\left(s_{1}, 0\right),\left(s_{2}, 0\right),\left(s_{3}, 0.375\right),\left(s_{4}, 0.875\right),\left(s_{5}, 0\right.\right.$. $\left.875),\left(s_{6}, 0.375\right)\right\}$. Graphically, they are represented in Fig. 3.

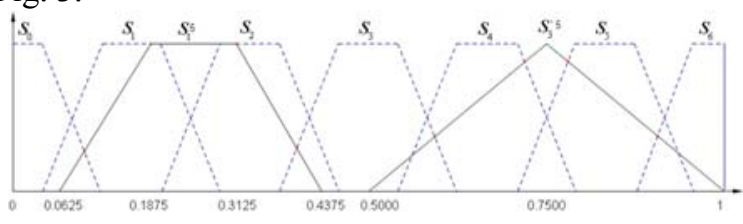

Fig. 3. The results of $s_{1}^{5}$ and $s_{3}^{\prime 5}$ are transformed into a fuzzy set in $S_{T}$.

It is clear that the heterogeneous information is transformed as a fuzzy set on a BLTS. These fuzzy sets are difficult to manage and hard to understand by DMs. So, in order to simplify the computations and improve the comprehensibility of the results obtained in this phase, we shall transform the collective values expressed by means of fuzzy sets on the BLTS into continues linguistic in the BLTS as:

$$
\chi\left(\tau_{H S_{T}}(H)\right)=\left(\sum_{i=0}^{\tau} \gamma_{i}\right)^{-1}\left(\bigoplus_{i=0}^{\tau} \gamma_{i} s_{i}\right), s_{i} \in \bar{S}_{T} .
$$

\section{(3) Aggregate all DMs' opinions}

The collective assessments of alternatives against each criteria $x_{i j}$ can be calculated as ${ }^{3}$ :

$x_{i j}=1 / K \times\left(x_{i j}^{1} \oplus x_{i j}^{2} \oplus \ldots \oplus x_{i j}^{k}\right) i=1, \ldots, m, j=1, \ldots, n$.

(4) Determine the positive ideal solution and negative ideal solution.

The positive ideal solution $A^{+}$consists of all of best values attainable of criteria.

$$
\begin{aligned}
A^{+} & =\left\{x_{.1}^{+}, \ldots, x_{. n}^{+}\right\} \\
& =\left\{\left(\max _{i} x_{i j}, j \in \Omega_{b}\right),\left(\min _{i} x_{i j}, j \in \Omega_{c}\right)\right\} .
\end{aligned}
$$

The negative ideal solution $A^{-}$is composed of all worst values attainable of criteria

$$
\begin{aligned}
A^{-} & =\left\{x_{.1}^{-}, \ldots, x_{. n}^{-}\right\} \\
& =\left\{\left(\min _{i} x_{i j}, j \in \Omega_{b}\right),\left(\max _{i} x_{i j}, j \in \Omega_{c}\right)\right\} .
\end{aligned}
$$

where $\Omega_{b}, \Omega_{c}$ denote the benefit criteria set, and the cost criteria set, respectively.
(5) Calculate the separation measures of individual criteria.

The separation of each criteria from its positive ideal value is defined as

$$
d_{i j}^{+}=d\left(x_{j}^{+}, x_{i j}\right) .
$$

Similarly, the separation of each criteria from its negative ideal value is given as

$$
d_{i j}^{-}=d\left(x_{j}^{-}, x_{i j}\right) .
$$

(6) Calculate the overall separation measures of alternatives.

In general, the overall separation measures of alternatives are measured by distance measures, which usually using the weighted hamming distance (WHD), the weighted euclidean distance(WED), the weighted minkowski distance(WMD) or the weighted chebyshev distance(WCD), etc. They simply compute the distance measures without considering the DMs' attitudes. However, in some cases, the decision is assumed to be completely dependent on DMs in a decision process. Therefore, the DMs' attitudes can be considered as an important factor in making suitable decision. In other words, sometimes DMs are interesting to consider the possibility of parameterizing the results from the maximum distance to the minimum distance.

The OWA operator ${ }^{20}$ for aggregating values (e.g., "satisfaction levels" with respect to criteria) was introduced by Yager (1988), which more explicitly recognize the "attitudinal character" of the decisionmakers by using linguistic quantifier, such as: "at least some criteria must be taken into account". The OWA operator enables to move continuously from logical "and" to logical "or" i.e., from non-compensatory to full-compensatory situation.

Definition $7^{20}$. An ordered weighted averaging (OWA) operator of dimension $n$ is a mapping OWA : $R^{n} \rightarrow R$, that has an associated $n$ vector $W: w_{j} \geq 0, j=1,2, \ldots, n$ and $\sum_{j=1}^{n} w_{j}=1$, which has the following form:

$$
O W A\left(\alpha_{1}, \alpha_{2}, \ldots, \alpha_{n}\right)=\sum_{j=1}^{n} w_{j} \beta_{j} .
$$

where $\beta_{j}$ is the $j$ th largest of the arguments $\alpha_{j}(j=1,2, \ldots, n)$.

Definition 8. Let $S^{a}=\left(s_{1}^{a}, \ldots, s_{n}^{a}\right)$ and $S^{b}=\left(s_{1}^{b}, \ldots, s_{n}^{b}\right)$ be two collections of linguistic arguments, then an ordered weighted distance (OWD) measure is defined as: 


$$
O W D\left(S^{a}, S^{b}\right)=\sum_{j=1}^{n} w_{j} d_{\sigma(j)}
$$

where $d_{j}=d\left(s_{j}^{a}, s_{j}^{b}\right)$ be the distance between $s_{j}^{a}$ and $s_{j}^{b}$, and is calculated by Eq. (4); $\sigma(\cdot)$ is any permutation of $(1, \ldots, n)$ such that $\sigma(j-1) \geq \sigma(j), j=2, \ldots, n$, and $w=\left(w_{1}, \ldots, w_{n}\right)$ is the weighting vector of the ordered values of the differences, with $w_{j} \geq 0, j=1, \ldots, n$ and $\sum_{j=1}^{n} w_{j}=1$.

Similar to OWA operator, the fundamental aspect of the OWD measure is the reordering step. From a generalized perspective of the reordering step, it is possible to distinguish between the descending OWD (DOWD) measure and the ascending OWD (AOWD) measure.

To apply the OWD measure (or OWA operator) to decision making, a crucial issue is to determine its weight vector. Different approaches have been suggested for obtaining the weight vector ${ }^{21,22}$. The most common approach is the one based on the use of a linguistic quantifier ${ }^{21}, \mathrm{Q}$, which is used to indicate the portion of the criteria DMs feel is necessary for a good solution. The linguistic quantifier allows DMs to translate their decision attitudes with a linguistic term such as "most" or "many". The OWD measure can adjust the weight of a criteria based on the attitudes of DMs. Yager $(1996)^{21}$ recommended obtaining the weights of OWA operator as follows:

$$
w_{j}=Q\left(\frac{j}{n}\right)-Q\left(\frac{j-1}{n}\right), j=1, \ldots, n .
$$

The simplest and most commonly quantifier, Q, is defined as:

$$
Q(r)=r^{\alpha}, \alpha \geq 0, r \in[0,1] .
$$

The decision attitude is specified with parameter $\alpha$, its changes represent a continuum of different decision attitudes between the two extreme cases of requiring "at least one" and "all" the criteria to be satisfied. The common decision attitudes and their corresponding parameter $\alpha$ are as following ${ }^{23}$ :

$\begin{array}{ll}\text { - All (AND operator) } & \alpha \rightarrow \infty:(1000) \\ \text { - Most } & \alpha=5 \\ \text { - Many } & \alpha=2 \\ \text { - Half (mean operator) } & \alpha=1 \\ \text { - Some } & \alpha=0.5 \\ \text { - Few } & \alpha=0.2\end{array}$

- At least one (OR operator) $\quad \alpha \rightarrow 0:(0.001)$

By combining (18),(19) and (20), we have:

$\operatorname{OWD}\left(S^{a}, S^{b}\right)=\sum_{j=1}^{n}\left[\left(\frac{j}{n}\right)^{\alpha}-\left(\frac{j-1}{n}\right)^{\alpha}\right] d_{\sigma(j)}$.

The OWD measure is capable of synthesizing the linguistic information in consideration with the DMs' attitudes. So it expresses directly the intensity of compromise or the type of compromise wanted by the DMs.

According to the basic principle of the TOPSIS, the shorter distance of alternative to the positive ideal solution and the farther distance of alternative to the negative ideal solution, the better alternative is. In this paper, the overall separation measures of alternatives to positive solution are defined according to AOWD as:

$$
\zeta_{i}^{+}=\sqrt{\sum_{j=1}^{n}\left(w_{j} d_{i \sigma(j)}^{+}\right)^{2}},(i=1, \ldots, m) .
$$

being $\sigma:\{1,2, \ldots, n\} \rightarrow\{1,2, \ldots, n\}, \forall j=1,2, \ldots, n-1$, i.e., $d_{i \sigma(j)}^{+}$is the $j$ th smallest value in the set $\left\{d_{i \sigma(1)}^{+}, d_{i \sigma(2)}^{+}, \ldots, d_{i \sigma(n)}^{+}\right\}$.

The overall separation measures of alternatives to negative solution based on DOWD are defined as:

$$
\zeta_{i}^{-}=\sqrt{\sum_{j=1}^{n}\left(w_{j} d_{i \sigma(j)}^{-}\right)^{2}},(i=1, \ldots, m) .
$$

being $\sigma:\{1,2, \ldots, n\} \rightarrow\{1,2, \ldots, n\}, \forall j=1,2, \ldots, n-1$, i.e., $d_{i \sigma(j)}^{-}$is the $j$ th largest value in the set $\left\{d_{i \sigma(1)}^{-}, d_{i \sigma(2)}^{-}, \ldots, d_{i \sigma(n)}^{-}\right\}$.

(7) Calculate the relative closeness of each alternative to the ideal solutions.

$$
\tilde{C}_{i}=\varsigma_{i}^{-} \phi\left(\varsigma_{i}^{+} \oplus \varsigma_{i}^{-}\right), i=1, \ldots, m .
$$

(8) Rank the alternatives according to the relative closenesses. The bigger the $\tilde{C}_{i}$, the better the alternative $A_{i}$.

In sum, the multi-criteria group decision making with heterogeneous information based on ideal points concept can be summed up as follows:

- Construct decision matrices $D_{k}, k=1, \ldots, K$. Decision makers provide their assessments with their own preferred representation formats, and decide their decision attitude according to real decision situation.

- $\quad$ Select basic linguistic term set $S_{T}$. 
- Calculate the normalized numerical values $x_{i j}$ by Eq.(5)and interval values $\left[x_{i j}^{L}, x_{i j}^{R}\right]$ by Eqs. (6) and (7), respectively.

- Unify heterogeneous information by Eqs.(10) and (11).

- $\quad$ Aggregate all DMs' opinions by Eq.(12).

- Determine the positive ideal solution $A^{+}$and negative ideal solution $A^{-}$by Eqs.(13) and (14), respectively.

- Calculate the separation measures of individual criteria $d_{i j}^{+}$and $d_{i j}^{-}$by Eqs.(15) and (16), respectively.

- Determine weights of criteria by Eqs.(19) and (20).

- Calculate the overall separation measures of alternatives $\varsigma_{i}^{+}$and $\varsigma_{i}^{-}$by Eqs.(22) and (23).

- Rank the alternatives according to the relative closenesses $\tilde{C}_{i}$ by Eq.(24).

\section{Numerical example}

In this section, an example is used to illustrate the use of proposed method. We consider extending the linguistic information used in the numerical example from Ref.3 to the form of heterogeneous information. Three candidates $A_{1}, A_{2}$ and $A_{3}$ remain for further evaluation. A committee of five decision-makers, $D_{1}, D_{2}, D_{3}, D_{4}$ and $D_{5}$ has been formed to conduct the interview and to select the most suitable candidate. Five benefit criteria are considered:

(1) emotional steadiness $\left(C_{1}\right)$,

(2) oral communication skill $\left(C_{2}\right)$,

(3) personality $\left(C_{3}\right)$,

(4) past experience $\left(C_{4}\right)$,

(5) self-confidence $\left(C_{5}\right)$.

The proposed method is currently applied to solve this problem and the computational procedure is summarized as follows:

(1) According to these DMs' preferences, they use different formats information to represent their opinions over the alternatives set as following:

$D_{1}$ provides his assessments in the linguistic term set with 5 labels represented by trapezoidal fuzzy number: $\left\{s_{0}, s_{1}, s_{2}, s_{3}, s_{4}\right\}=\{\mathrm{Bad}$, Poor, Fair, Good, Perfect\}

$D_{2}$ provides his assessments in the linguistic term set with 7 labels represented by trapezoidal fuzzy number: $\left\{s_{0}, s_{1}, s_{2}, s_{3}, s_{4}, s_{5}, s_{6}\right\}=\{\mathrm{Bad}$, Very Poor, Poor, Fair, Good, Very Good, Perfect\}
$D_{3}$ provides his assessments in linguistic term set with 5 labels represented by triangular fuzzy number: $\left\{s_{0}, s_{1}, s_{2}, s_{3}, s_{4}\right\}=\{$ Bad, Poor, Fair, Good, Perfect $\}$

$D_{4}$ provides his preferences with interval number in $[0,1]$ interval.

$D_{5}$ provides his assessments with real number in $[0,1]$ interval.

The assessments of the three alternatives under all criteria are provided by DMs as follows:

$$
\begin{aligned}
& D^{1}=\left[\begin{array}{lllll}
s_{3} & s_{2} & s_{3} & s_{4} & s_{2} \\
s_{3} & s_{4} & s_{4} & s_{4} & s_{2} \\
s_{3} & s_{4} & s_{2} & s_{4} & s_{3}
\end{array}\right], D^{2}=\left[\begin{array}{lllll}
s_{4} & s_{5} & s_{3} & s_{6} & s_{3} \\
s_{5} & s_{6} & s_{6} & s_{6} & s_{6} \\
s_{6} & s_{4} & s_{5} & s_{5} & s_{5}
\end{array}\right], \\
& D^{3}=\left[\begin{array}{lllll}
s_{3} & s_{2} & s_{3} & s_{4} & s_{2} \\
s_{3} & s_{4} & s_{3} & s_{4} & s_{2} \\
s_{2} & s_{4} & s_{4} & s_{3} & s_{2}
\end{array}\right], D^{5}=\left[\begin{array}{llllll}
0.7 & 0.8 & 0.6 & 0.9 & 0.5 \\
0.8 & 1.0 & 0.9 & 0.7 & 0.9 \\
0.9 & 0.7 & 0.8 & 0.9 & 0.8
\end{array}\right],
\end{aligned}
$$

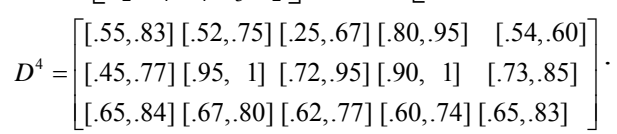

In order to verify the proposed method, in the case, we adopt to seven common decision attitudes to compare analysis.

(2)Select basic linguistic term set. According to the granularity, the linguistic terms set of 7 labels represented by trapezoidal fuzzy number severed as BLTS.

In the case, the criteria evaluated with numerical and interval valued information are in $[0,1]$ interval, so the sub-steps that normalize numerical and interval valued criteria values without consideration.

(3) Unify heterogeneous information by using Eqs.(10) and (11), the unified information and the ones represented by continuous terms set in BLTS of each criteria with respect to each alternative provided by each DM are shown in Table1-5, respectively. In the sequel, OI, $\mathrm{HI}$ and $\mathrm{CL}$ denote original information, homogenized information, and the continuous linguistic sets represented information, respectively.

Table 1. Unified results of linguistic values in the set with 5 labels represented by trapezoidal fuzzy number.

\begin{tabular}{cll}
\hline OI & \multicolumn{1}{c}{ HI } & CL \\
\hline $\mathrm{s}_{3}$ & $\left(\mathrm{~s}_{3}, 0.3\right),\left(\mathrm{s}_{4}, 1\right),\left(\mathrm{s}_{5}, 1\right),\left(\mathrm{s}_{6}, 0.3\right)$ & $\mathrm{s}_{4.5}$ \\
$\mathrm{~s}_{2}$ & $\left(\mathrm{~s}_{2}, 0.7\right),\left(\mathrm{s}_{3}, 1\right),\left(\mathrm{s}_{4}, 0.7\right)$ & $\mathrm{s}_{3.4}$ \\
$\mathrm{~s}_{4}$ & $\left(\mathrm{~s}_{5}, 0.7\right),\left(\mathrm{s}_{6}, 1\right)$ & $\mathrm{s}_{5.6}$ \\
\hline
\end{tabular}


Table 2. Unified results of linguistic values in the set with 5 labels represented by triangular fuzzy number.

\begin{tabular}{lll}
\hline OI & \multicolumn{1}{c}{ HI } & CL \\
\hline $\mathrm{s}_{3}$ & $\left(\mathrm{~s}_{3}, 0.375\right),\left(\mathrm{s}_{4}, 0.875\right),\left(\mathrm{s}_{5}, 0.875\right),\left(\mathrm{s}_{6}, 0.375\right)$ & $\mathrm{s}_{4.5}$ \\
$\mathrm{~s}_{2}$ & $\left(\mathrm{~s}_{1}, 0.125\right),\left(\mathrm{s}_{2}, 0.625\right),\left(\mathrm{s}_{3}, 1\right),\left(\mathrm{s}_{4}, 0.625\right),\left(\mathrm{s}_{5}, 0.125\right)$ & $\mathrm{s}_{3}$ \\
$\mathrm{~s}_{4}$ & $\left(\mathrm{~s}_{4}, 0.125\right),\left(\mathrm{s}_{5}, 0.625\right),\left(\mathrm{s}_{6}, 0.750\right)$ & $\mathrm{s}_{5.4}$ \\
\hline
\end{tabular}

Table 4. Unified results of interval values.

\begin{tabular}{cll}
\hline $\mathbf{O I}$ & \multicolumn{1}{c}{ HI } & $\mathbf{C L}$ \\
\hline$[0.55,0.83]$ & $\left(\mathrm{s}_{3}, 0.9\right),\left(\mathrm{s}_{4}, 1\right),\left(\mathrm{s}_{5}, 1\right)$ & $\mathrm{s}_{3.9}$ \\
{$[0.52,0.75]$} & $\left(\mathrm{s}_{3}, 1\right),\left(\mathrm{s}_{4}, 1\right),\left(\mathrm{s}_{5}, 0.5\right)$ & $\mathrm{s}_{3.8}$ \\
{$[0.25,0.67]$} & $\left(\mathrm{s}_{1}, 0.5\right),\left(\mathrm{s}_{2}, 1\right),\left(\mathrm{s}_{3}, 1\right),\left(\mathrm{s}_{4}, 1\right)$ & $\mathrm{s}_{2.7}$ \\
{$[0.80,0.95]$} & $\left(\mathrm{s}_{5}, 1\right),\left(\mathrm{s}_{6}, 0.9\right)$ & $\mathrm{s}_{5.5}$ \\
{$[0.54,0.60]$} & $\left(\mathrm{s}_{3}, 1\right),\left(\mathrm{s}_{4}, 1\right),\left(\mathrm{s}_{5}, 0.74\right)$ & $\mathrm{s}_{3.9}$ \\
{$[0.45,0.77]$} & $\left(\mathrm{s}_{2}, 0.1\right),\left(\mathrm{s}_{3}, 1\right),\left(\mathrm{s}_{4}, 1\right),\left(\mathrm{s}_{5}, 0.74\right)$ & $\mathrm{s}_{3.8}$ \\
{$[0.95,1]$} & $\left(\mathrm{s}_{5}, 0.1\right),\left(\mathrm{s}_{6}, 1\right)$ & $\mathrm{s}_{5.9}$ \\
{$[0.72,0.95]$} & $\left(\mathrm{s}_{4}, 0.86\right),\left(\mathrm{s}_{5}, 1\right),\left(\mathrm{s}_{6}, 0.9\right)$ & $\mathrm{s}_{5}$ \\
{$[0.90,1]$} & $\left(\mathrm{s}_{5}, 0.7\right),\left(\mathrm{s}_{6}, 1\right)$ & $\mathrm{s}_{5.6}$ \\
{$[0.73,0.85]$} & $\left(\mathrm{s}_{4}, 0.83\right),\left(\mathrm{s}_{5}, 1\right)$ & $\mathrm{s}_{4.5}$ \\
{$[0.65,0.84]$} & $\left(\mathrm{s}_{4}, 1\right),\left(\mathrm{s}_{5}, 1\right)$ & $\mathrm{s}_{4.5}$ \\
{$[0.67,0.80]$} & $\left(\mathrm{s}_{4}, 1\right),\left(\mathrm{s}_{5}, 1\right)$ & $\mathrm{s}_{4.5}$ \\
{$[0.62,0.77]$} & $\left(\mathrm{s}_{3}, 0.06\right),\left(\mathrm{s}_{4}, 1\right),\left(\mathrm{s}_{5}, 0.74\right)$ & $\mathrm{s}_{4.4}$ \\
{$[0.60,0.74]$} & $\left(\mathrm{s}_{3}, 0.3\right),\left(\mathrm{s}_{4}, 1\right),\left(\mathrm{s}_{5}, 0.38\right)$ & $\mathrm{s}_{4}$ \\
{$[0.65,0.83]$} & $\left(\mathrm{s}_{4}, 1\right),\left(\mathrm{s}_{5}, 1\right)$ & $\mathrm{s}_{4.5}$ \\
\hline
\end{tabular}

Table 5. Unified results of crisp values.

\begin{tabular}{cll}
\hline OI & \multicolumn{1}{c}{ HI } & CL \\
\hline 0.7 & $\left(\mathrm{~s}_{4}, 1\right)$ & $\mathrm{s}_{4}$ \\
0.8 & $\left(\mathrm{~s}_{5}, 1\right)$ & $\mathrm{s}_{5}$ \\
0.6 & $\left(\mathrm{~s}_{3}, 0.3\right),\left(\mathrm{s}_{4}, 0.7\right)$ & $\mathrm{s}_{3.7}$ \\
0.9 & $\left(\mathrm{~s}_{5}, 0.7\right),\left(\mathrm{s}_{6}, 0.3\right)$ & $\mathrm{s}_{5.3}$ \\
0.5 & $\left(\mathrm{~s}_{3}, 1\right)$ & $\mathrm{s}_{3}$ \\
1 & $\left(\mathrm{~s}_{6}, 1\right)$ & $\mathrm{s}_{6}$ \\
\hline
\end{tabular}

(4)The collective assessments of alternatives against each criteria are calculated by using Eq.(12), the results are shown in Table. 6.

Table 6 The collective assessments of criteria versus alternatives.

\begin{tabular}{cccccc}
\hline & $\mathrm{C}_{1}$ & $\mathrm{C}_{2}$ & $\mathrm{C}_{3}$ & $\mathrm{C}_{4}$ & $\mathrm{C}_{5}$ \\
\hline $\mathrm{A}_{1}$ & $\mathrm{~s}_{4.18}$ & $\mathrm{~s}_{4.04}$ & $\mathrm{~s}_{3.68}$ & $\mathrm{~s}_{5.56}$ & $\mathrm{~s}_{3.56}$ \\
$\mathrm{~A}_{2}$ & $\mathrm{~S}_{4.58}$ & $\mathrm{~s}_{5.78}$ & $\mathrm{~S}_{5.28}$ & $\mathrm{~s}_{5.72}$ & $\mathrm{~S}_{4.44}$ \\
$\mathrm{~A}_{3}$ & $\mathrm{~s}_{4.66}$ & $\mathrm{~s}_{4.7}$ & $\mathrm{~s}_{4.64}$ & $\mathrm{~S}_{4.88}$ & $\mathrm{~s}_{4.4}$ \\
\hline
\end{tabular}

(5) Determine ideal points by using Eqs.(13) and (14), the positive and negative ideal solutions are shown in Table 7.

(6) The separation measures of individual criteria are obtained by Eqs.(15) and (16), respectively. The separation measures of individual criteria to their positive ideal points and negative ideal points against alternatives are listed in Table 8 and Table 9, respectively.
Table 7. The positive/negative ideal solutions.

\begin{tabular}{lcc}
\hline & Positive ideal point & Negative ideal point \\
\hline $\mathrm{C}_{1}$ & $x_{1}^{+}=\mathrm{s}_{4.66}$ & $x_{1}^{-}=\mathrm{s}_{4.18}$ \\
$\mathrm{C}_{2}$ & $x_{2}^{+}=\mathrm{s}_{5.78}$ & $x_{2}^{-}=\mathrm{s}_{4.04}$ \\
$\mathrm{C}_{3}$ & $x_{3}^{+}=\mathrm{s}_{5.28}$ & $x_{3}^{-}=\mathrm{s}_{3.68}$ \\
$\mathrm{C}_{4}$ & $x_{4}^{+}=\mathrm{s}_{5.72}$ & $x_{4}^{-}=\mathrm{s}_{4.88}$ \\
$\mathrm{C}_{5}$ & $x_{5}^{+}=\mathrm{s}_{4.44}$ & $x_{5}^{-}=\mathrm{s}_{3.56}$ \\
\hline
\end{tabular}

Table 8 . The separation measures of individual criteria to their positive ideal solution.

\begin{tabular}{cccccc}
\hline & $\mathrm{C}_{1}$ & $\mathrm{C}_{2}$ & $\mathrm{C}_{3}$ & $\mathrm{C}_{4}$ & $\mathrm{C}_{5}$ \\
\hline $\mathrm{A}_{1}$ & 0.48 & 1.74 & 1.60 & 0.16 & 0.88 \\
$\mathrm{~A}_{2}$ & 0.08 & 0 & 0 & 0 & 0 \\
$\mathrm{~A}_{3}$ & 0 & 1.08 & 0.64 & 0.84 & 0.04 \\
\hline
\end{tabular}

Table 9. The separation measures of individual criteria to their negative ideal solution.

\begin{tabular}{cccccc}
\hline & $\mathrm{C}_{1}$ & $\mathrm{C}_{2}$ & $\mathrm{C}_{3}$ & $\mathrm{C}_{4}$ & $\mathrm{C}_{5}$ \\
\hline $\mathrm{A}_{1}$ & 0 & 0 & 0 & 0.68 & 0 \\
$\mathrm{~A}_{2}$ & 0.4 & 1.73 & 1.60 & 0.64 & 0.88 \\
$\mathrm{~A}_{3}$ & 0.48 & 0.66 & 0.96 & 0 & 0.84 \\
\hline
\end{tabular}

(7) The ordered weights are determined by Eqs.(19) and (20). The weights under the common decision attitudes are listed in Table 10.

Table 10. The weights under the common decision attitudes.

\begin{tabular}{lllllll}
\hline $\begin{array}{l}\text { Decision } \\
\text { attitude }\end{array}$ & $\alpha$ & $w_{1}$ & $w_{2}$ & $w_{3}$ & $w_{4}$ & $w_{5}$ \\
\hline All & 1000 & 0.000 & 0.000 & 0.000 & 0.000 & 1.000 \\
Most & 5 & 0.000 & 0.010 & 0.068 & 0.250 & 0.672 \\
Many & 2 & 0.040 & 0.120 & 0.200 & 0.280 & 0.360 \\
Half & 1 & 0.200 & 0.200 & 0.200 & 0.200 & 0.200 \\
Some & 0.5 & 0.447 & 0.185 & 0.143 & 0.119 & 0.106 \\
Few & 0.2 & 0.725 & 0.108 & 0.0 .70 & 0.053 & 0.044 \\
At least one & 0.00001 & 1.000 & 0.000 & 0.000 & 0.000 & 0.000 \\
\hline
\end{tabular}

(8) Calculate the overall separation measures of alternatives from the positive and negative ideal solutions by Eqs.(22) and (23), respectively. The overall separation measures of alternatives from the positive ideal solutions and negative ideal solutions are listed in Table 11 and Table 12, respectively.

Table 11. The overall separation measures of alternatives from the positive ideal solution.

\begin{tabular}{cccccccc}
\hline & All & Most & Many & Half & Some & Few & At least one \\
\hline $\mathrm{A}_{1}$ & 1.74 & 1.63 & 1.31 & 0.97 & 0.66 & 0.39 & 0.16 \\
$\mathrm{~A}_{2}$ & 0.08 & 0.05 & 0.03 & 0.02 & 0.01 & 0 & 0 \\
$\mathrm{~A}_{3}$ & 1.08 & 0.98 & 0.76 & 0.52 & 0.31 & 0.14 & 0 \\
\hline
\end{tabular}


Table 12. The overall separation measures of alternatives from the negative ideal solution.

\begin{tabular}{cccccccc}
\hline & All & Most & Many & Half & Some & Few & At least one \\
\hline $\mathrm{A}_{1}$ & 0.68 & 0 & 0.03 & 0.14 & 0.30 & 0.49 & 0.68 \\
$\mathrm{~A}_{2}$ & 1.73 & 0.50 & 0.76 & 1.05 & 1.31 & 1.54 & 1.73 \\
$\mathrm{~A}_{3}$ & 0.96 & 0.17 & 0.41 & 0.59 & 0.74 & 0.86 & 0.96 \\
\hline
\end{tabular}

(9) The overall relative closenesses of alternatives are calculated by Eq.(24). The final results under the seven situations are shown in Table 13, and they are represented graphically in Fig. 4.

Table 13. The overall relative closenesses under different decision attitudes.

\begin{tabular}{cccccccc}
\hline & All & Most & Many & Half & Some & Few & At least one \\
\hline $\mathrm{A}_{1}$ & 0.28 & 0 & 0.02 & 0.12 & 0.31 & 0.56 & 0.81 \\
$\mathrm{~A}_{2}$ & 0.96 & 0.91 & 0.96 & 0.98 & 0.99 & 1 & 1 \\
$\mathrm{~A}_{3}$ & 0.47 & 0.15 & 0.35 & 0.53 & 0.70 & 0.86 & 1 \\
\hline
\end{tabular}

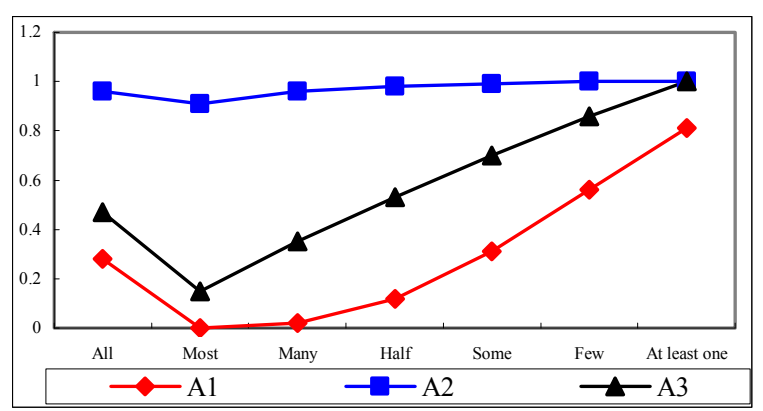

Fig. 4. Comparison of overall relative closenesses under different decision attitudes.

The above results indicate that the final ranks all are $x_{2} \succ x_{3} \succ x_{1}$, which imply the proposed TOPSIS method can provide robust results under different decision situations. In addition, these ranking results that obtained from the group of $\mathrm{DMs}$ and $\mathrm{DM}_{5}$ are consistent have even more support the proposed method. Moreover, we extend the Deng et al.' ${ }^{24}$ TOPSIS method, and the MCDM method of Yager ${ }^{20}$ to our heterogeneous information context, then the ranking results calculated by the two methods are the same as the results calculated by the method proposed, so it is demonstrated that the method proposed in this paper is feasible and effective.

\section{Conclusions}

Owing to the facts that the decision information provide by decision makers can be of a diversity nature, and decision making in different decision situations needing different decision strategies. In the paper, we proposed a more flexible multiple criteria group decision making method based on ideal points concept, which not only allows DMs represent their assessments in different representation formats, such as real values, interval values and linguistic values, but also offers DMs the possibility of expressing their decision attitudes according to their interests and the nature of decision problem. In the proposed method, the heterogeneous information is homogenized as a fuzzy set on a predefined basic linguistic terms set (BLTS), then the homogenized information is further transformed to continuous linguistic terms in the BLTS to simplify the computations and improve the comprehensibility. The separation measure based on ordered weighted distance is introduced to reflect the different decision attitudes of DMs according to their interests and real decision situations. The proposed method is effective and feasible just as shown in the illustrative example. It is expected that the proposed method can be applied to the fields of supply chain management, mobile-business.

\section{Acknowledgements}

The authors are very grateful to the Editor, Professor Javier Montero, and the anonymous referees for their insightful and constructive comments and suggestions which have helped to improve the paper. This work was supported in part by the National Natural Science Funds of China (Project Nos.: 71272191 and 71072085), the Graduate Innovation Funds of Heilongjiang Province of China (Project No.: YJSCX2011-003HLJ), and the Educational Commission of Heilongjiang Province of China(Project No.: 11522Z031).

\section{References}

1. C.L. Hwang and K. Yoon, Multiple Attribute Decision Making (Springer-Verlag, Berlin, 1981).

2. H.-S. Shih, H.-J. Shyur and E. S. Lee, An extension of TOPSIS for group decision making, Mathematical and Computer Modelling 45 (2007) 801-813.

3. C.-T. Chen, Extensions of the TOPSIS for group decision-making under fuzzy environment, Fuzzy Sets and Systems 114 (2000) 1-9.

4. G.R. Jahanshahloo, F.H. Lotfi and M. Izadikhah, An algorithmic method to extend TOPSIS for decisionmaking problems with interval data, Applied Mathematics and Computation 175 (2006) 1375-1384.

5. Y.-M., Wang and T.M.S. Elhag, Fuzzy TOPSIS method based on alpha level sets with an application to bridge 
risk assessment, Expert Systems with Applications 31 (2006) 309-319.

6. Y.-J. Wang and H.-S. Lee, Generalizing TOPSIS for fuzzy multiple-criteria group decision-making, Computers and Mathematics with Applications 53 (2007) 1762-1772.

7. M.-F. Chen and G.-H. Tzeng, Combining grey relation and TOPSIS concepts for selecting an expatriate host country, Mathematical and Computer Modelling $\mathbf{4 0}$ (2004) 1473-1490.

8. M.S. García-Cascales and M.T. Lamata, Solving a decision problem with linguistic information, Pattern Recognition Letters 28 (2007) 2284-2294.

9. S.-M. Chen and L.-W. Lee, Fuzzy multiple attributes group decision-making based on the interval type-2 TOPSIS method, Expert Systems with Applications 37 (2010) 2790-2798.

10. D.-F. Li, TOPSIS based nonlinear programming methodology for MADM with interval-valued intuitionistic fuzzy sets, IEEE Transactions on Fuzzy Systems 18(2) (2010) 299-311.

11. Y.-C.Hu, Classification performance evaluation of single-layer perceptron with Choquet integral-based TOPSIS, Appl. Intell. 29 (2008) 204-215.

12. S.H. Zanakis, A. Solomon, N. Wishart, and S. Dublish, Multi-attribute decision making: A simulation comparison of selection methods, European Journal of Operational Research 107 (1998) 507-529.

13. L.A. Zadeh, The concept of a linguistic variable and its applications to approximate reasoning. Part I, Information Sciences 8 (1975) 199-249.

14. F. Herrera and E. Herrera-Viedma, Linguistic decision analysis: steps for solving decision problems under linguistic information, Fuzzy Sets and Systems 115 (2000) $67-82$.

15. Z. Xu, A method based on linguistic aggregation operators for group decision making with linguistic preference relations, Information Sciences 166 (2004) 19-30.

16. Z. Xu, A method for multiple attribute decision making with incomplete weight information in linguistic setting, Knowledge-Based Systems 20 (2007) 719-725.

17. F. Herrera, E. Herrera-Viedma and L. Martinez, A fusion approach for managing multi-granularity linguistic term sets in decision making, Fuzzy Sets and Systems 114 (2000) 43-58.

18. F. Herrera, L. Martinez and P.J. Sanchez, Managing nonhomogeneous information in group decision making, European Journal of Operational Research 166 (2005) $115-132$

19. L. Martinez, J. Liu, D. Ruan and J.-B. Yang, Dealing with heterogeneous information in engineering evaluation processes, Information Sciences 177 (2007) 1533-1542.

20. R.R. Yager, On ordered weighted averaging aggregation operators in multicriteria decision-making, IEEE
Transactions on Systems, Man, and Cybernetics 18(1) (1988) 183-190.

21. R.R. Yager, Quantifier guided aggregation using OWA operators, International Journal Intelligent System 11(1) (1996) 49-73.

22. Z. Xu, An overview of methods for determining OWA weights, International Journal of Intelligent Systems $\mathbf{2 0}$ (2005) 843-865.

23. J. Malczewski, Ordered weighted averaging with fuzzy quantifiers: GIS-based multicriteria evaluation for landuse suitability analysis, International Journal of Applied Earth Observation and Geoinformation 8 (2006) 270277.

24. H. Deng, C.-H. Yeh and R.J. Willis, Inter-company comparison using modified TOPSIS with objective weights, Computers \& Operations Research 27 (2000) 963-973. 